\title{
The Analysis and Probabilistic Health Risk Assessment of Polycyclic Aromatic Hydrocarbons Contamination in Vegetables and Fruits Samples Marketed Tehran With Chemometric Approach
}

\author{
Fariba Khalili \\ Department of Environmental Health Engineering, School of Public Health, Tehran University of Medical Sciences, Tehran \\ Nabi Shariatifar ( $\square$ nshariati@tums.ac.ir) \\ Department of Environmental Health Engineering, School of Public Health, Tehran University of Medical Sciences, Tehran \\ Mohammad Hadi Dehghani \\ Department of Environmental Health Engineering, School of Public Health, Tehran University of Medical Sciences, Tehran \\ Kamyar Yaghmaeian \\ Department of Environmental Health Engineering, School of Public Health, Tehran University of Medical Sciences, Tehran \\ Ramin Nabizadeh Nodehi \\ Department of Environmental Health Engineering, School of Public Health, Tehran University of Medical Sciences, Tehran \\ Mehdi Yaseri \\ Department of Epidemiology and Biostatistics, School of Public Health, Tehran University of Medical Sciences, Tehran
}

\section{Research Article}

Keywords: Health risk assessment, PAHs, Vegetables, Fruits, GC-MS

Posted Date: December 23rd, 2020

DOl: https://doi.org/10.21203/rs.3.rs-130120/v1

License: (c) (i) This work is licensed under a Creative Commons Attribution 4.0 International License. Read Full License 


\section{Abstract}

The aim of current study was to evaluate the polycyclic aromatic hydrocarbons (PAHs) concentration and probabilistic health risk in vegetables and fruits samples of Tehran city, Iran during 2018-2019 using magnetic solid-phase extraction (MSPE) and gas chromatography-mass spectrometry (GC-MS). The limit of detection (LOD) and limit of quantitation (LOQ) ranged 0.040-0.084 and 0.121-0.253 $\mu \mathrm{g} / \mathrm{kg}$, respectively. The results showed that the highest PAH levels corresponded to acenaphthene $(135.1 \pm 7.1 \mu \mathrm{g} / \mathrm{kg})$ and naphthalene $(114.1 \pm 5.0 \mu \mathrm{g} / \mathrm{kg})$, whereas the lowest concentrations were those of Benzo(a)pyrene (not detected), Benzo(k)fluoranthene (not detected), Indeno(1,2,3-cd)pyrene (not detected), Benzo(b)fluoranthene (not detected) and Benzo(g,h,i)perylene (not detected). Hierarchical cluster analysis (HCA) and principal component analysis (PCA) were applied to evaluate the correlation between the type and amount of 16 PAHs with vegetables and fruits samples. The results of Monte Carlo Simulation (MCS) revealed that the mean of incremental lifetime cancer risk (ILCR) in vegetables and fruits is $5.2 \mathrm{E}-05$ and $7.7 \mathrm{E}-05$ higher than the acceptable risk level $\left(10^{-6}\right)$. Finally, the highest ILCR in fruits and vegetables was related to cucumber (5.1E-04) and tomato (4.3E-04), respectively. Therefore, monitoring the PAHs concentrations in both groups of vegetables and fruits is necessary.

\section{Introduction}

Polycyclic aromatic hydrocarbon (PAH) compounds are considered as pervasive contaminants that form a large group of stable organic compounds and classify the presence of 2 or more welded aromatic rings ${ }^{1}$. These compounds have a moderately low solubility in water, they are extremely high in fat and oil and are soluble in most organic solvents ${ }^{2}$. Some PAH compounds have mutagenic, carcinogenic and teratogenic and have been linked to some types of cancer in laboratory animals and also in human. Among 16PAHs introduced using the US Environmental Protection Agency (USEPA) as food contaminants, benzo(a)pyrene $(\mathrm{BaP})$ is found to be carcinogenic and is categorized in the 1 st group as carcinogenic to human using the IARC (International Agency for Research on Cancer) ${ }^{3}$. According to current regulations, BaP concentration in certain food and vegetable and fruit should not be more than $1 \mu \mathrm{g} / \mathrm{kg}{ }^{4}$. Food and food products are contaminated with PAH compounds through food production, cooking at home, and environmental sources, as well as water, soil, and air, can contaminate vegetable and fruit by entering in these food. Therefore PAH compounds are found as contaminants in some food including smoked food, seafood, milk and milk products, meat and products of meat, vegetables, cereals, fruits, coffee, tea, fats and oils ${ }^{5,6}$.

The important routes of contact to PAH compounds are inhalation, ingestion and contact of dermal, as well as a severe concern occurs about the PAHs contamination in food ${ }^{7}$. Furthermore, PAHs can enter raw and cooked food in a variety of ways. In cooked food, PAHs are made during the incomplete fuel of charcoal on the surface of the food (fat food), oil, and other organic matters or by direct contact of deep lipids with the heat of flame. Therefore, these compounds can be detected in matrices of complex containing a food variety ${ }^{8}$. Also, one of the main sources of raw foods such as vegetables and fruits is the environmental pathway related to the presence of PAH (water, soil and air) which can be important ${ }^{9}$. In addition, PAHs accumulation in vegetables and fruits relies on several factors such as the concentration of PAHs, soil characteristics, and the physiological characteristics of vegetables and fruits ${ }^{10}$. An important health concern about PAHs is related to their mutagenicity. Thus, activation of metabolic in cells to dioepoxides causes damage in DNA reproduction and mutation ${ }^{11}$. About 100 PAHs have been identified, most of which are formed in pyrolytic processes. According to the US-EPA, PAHs (major contaminant) in the food include naphthalene (NAP), fluorine (FLO), phenanthrene (PHE), acenaphthylene (ACY), acenaphthene (ACE), fluoranthene (FLA), anthracene (ANT), benz[a]anthracene (BaA), pyrene (PYR), benzo[b] fluoranthene (BbF), and chrysene (CHR). Further, other PAHs are benzo[g,h,i]perylene (BghiP), benzo[k]fluoranthene (BkF), benzo[a]pyrene (BaP), indeno[1,2,3-cd] Pyrene (IcdP), and dibenzo[a,h] anthracene (DahA) ${ }^{12}$. Among these compounds, three cases (i.e., $\mathrm{BaP}, \mathrm{BaA}$, and $\mathrm{DhA}$ ) are categorized as carcinogenic of probably for human (Group $2 \mathrm{~A}$ ) by the IARC ${ }^{13}$.

In most studies, BaP is frequently applied as a marker for PAHs in the food (12). Furthermore, the carcinogenic characteristic of PAHs is a great deal of concern. For calculate the harmfulness or carcinogenicity of PAHs, toxic equivalency factors are generally applied through BaP approximation for measuring the estimate of BaP equivalent doses. It should be noted that PAHs determinate in vegetables and fruits is particularly problematic since these foodstuffs comprise large quantities of co-extractives such as polyphenols (quercetin), fibers, sugars, minerals, vitamins, organic acids and pigments, in particularly high quantities of chlorophyll. Additionally, non-volatile matrix components may deposit at inlet of GC inlet and in the column of GC, duo to the formation of new active sites and thus a decrease in the signal of GC. Therefore, using an effectual method for the extracting and cleaning processes of vegetables and fruits is essential ${ }^{14}$. During the recent years, various methods of sample pretreatment have been used for PAHs separation and pre-concentration in vegetable and fruit samples including stir-bar sportive extraction (SBSE), solid-phase extraction (SPE), and solid-phase micro-extraction (SPME). One of the disadvantages of SPE is that adsorbents should be placed in an SPE cartridge whose task is difficult. In addition, the drawbacks of SBSE are memory effects and manual operation. Regarding SPME, adsorbents are detached from the phase of aqueous by centrifugation or clarification which might be consuming the time when dealing with great volumes of samples. Further, the SPME fibers are comparatively expensive and the coatings of the polymer are highly delicate and fragile

15. Based on magnetic nanoparticles, MSPE has lately emerged as a talented technique for preparation of sample. In the MSPE technique, adsorbents of magnetic are dispersed regularly and directly in the sample solution ${ }^{16-18}$. Furthermore, target compound is adsorbed on the adsorbent and thus is separated from the solution of sample by a magnet of external used out of the vessel of extraction. Therefore, PAH compounds are cleaned from the adsorbents of magnetic with a solvent of organic for further analysis. Finally, the eluted extracts are analyzed using gas or liquid chromatography (GC or HPLC) with different types of detectors $8,14,16$. Furthermore, the technique of MSPE can be joined with dispersion extraction such that rapid mass transfer is obtained because ofthe sufficient connection surface area between analyte and sorbent, that is practical and useful to quick equilibrium ${ }^{17,19}$. It is noteworthy that this

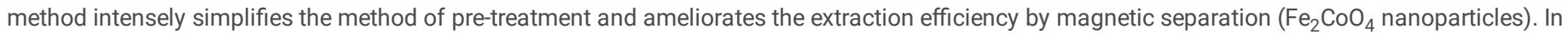
addition, magnetic adsorbents are recyclable. Therefore, MSPE offers some obvious benefits such as easiness and timesaving features, labor and cost. However, a limited body of research is available regarding measuring PAHs in vegetables and fruits by the MSPE technique ${ }^{15}$. The considering the relatively high proportion of vegetables and fruits in the food basket of Iran and other countries of the world, it is essential to test the PAHs level in vegetable and fruit. So, the aims of the current research are as follows: (1) to advance a simple, fast and reliable method for the analysis of PAHs in type of vegetables and fruits to eliminate the need of multi-phases column elution procedure using adsorbent of MSPE and GC/MS, (2) to assess the potential human health risk made by 
PAHs intake using the BaP cancer potency as a member of reference, and (3) finally, chemometric analysis was used to the correlation between PAHs in vegetable and fruits

\section{The Materials And Methods}

\subsection{Sample size}

In this study, 192 samples from 32 different vegetables and fruits were obtained from the markets of Tehran, Iran in May and December 2018 and February 2019. The vegetable samples included lettuce, cauliflower, white and purple cabbage, spinach, vegetable, eggplant, tomato, potato, onion, carrot, turnip, beet, and radish. Further, fruit samples encompassed sweet lemon, apple, banana, grape, orange, tangerine, grapefruit, persimmon, pomegranate, lemon, kiwi, watermelon, cucumber, cantaloupe, melon, peach, nectarine, and plum. Furthermore, a food frequency questionnaire was utilized to obtain the most consumed foods in Tehran. For this purpose, 700 questionnaires were randomly distributed among the adult group in the north, south, east, west, and center areas of Tehran, and some key parameters were obtained, including weight, age, and the ingestion amount of the intended food.

\subsection{Standards and reagents}

PAHs mix standards including 16 PAHs were bought from Supelco (Bellefonte, PA, U.S.). These compounds were NAP, FLO, PHE, ACY, ACE, FLA, ANT, BaA, YR, $\mathrm{BbF}, \mathrm{CHR}$, BghiP, BkF, BaP, IcdP, DahA. The solutions of standard were ready in dichloromethane, with $0.1 \mathrm{mg} / \mathrm{mL}$ concentration for all the above-mentioned PAHs. The stock standard solution was mixed with methanol-dichloromethane $(50: 50, \mathrm{v} / \mathrm{v})$ every week in order to make a working mixed solution $(1 \mu \mathrm{g} / \mathrm{mL}$ for each mentioned $\mathrm{PAH}$ ) which was used to measure the extraction function with various situations. Then, solutions of working and stock were preserved at $4{ }^{\circ} \mathrm{C}$, and biphenyl was used as the internal standard at a level of $0.05 \mu \mathrm{g} / \mathrm{mL}$ in methanol.

\subsection{Sample preparation and analysis}

The sample was prepared based on an important three-part procedure including sample clean-up, analyte adsorption, and analyte desorption from the adsorbent.

\section{a. Sample clean-up}

A five grams (vegetable and fruit) sample was weighed and one $\mathrm{mL}$ of the surrogate standard (biphenyl $0.05 \mathrm{mg} / \mathrm{mL}$ in $\mathrm{MeOH}$ ) was added, followed by adding $7.5 \mathrm{~mL} \mathrm{KOH} \mathrm{(1} \mathrm{molar)} \mathrm{and} 7.5 \mathrm{~mL}$ acetonitrile /methanol (30\%: v/v) and then homogenizing and sonicating the samples in an ultrasonic bath at $40{ }^{\circ} \mathrm{C}$ for $7 \mathrm{~min}$. Next, the prepared sample was centrifuged at $8944 \times \mathrm{g}$ for $15 \mathrm{~min}$, and the fat of each samples was then eliminated using the method of freezinglipid filtration ${ }^{20}$. Finally, the $\mathrm{pH}$ was adjusted with $\left.\mathrm{HCl}\right) 1 \mathrm{M}$ ) to 6.5 .

\section{b. Adsorption of analyte}

The water phase was moved to another vessel after the primary clean-up procedure. Then, $10 \mathrm{mg}$ of multi-walled carbon nanotube/CoFe2O4 (MWCNT/ CoFe204) composite (adsorbent) were prepared ${ }^{8}$ and 500 mg sodium chloride was added into the container. Next, the prepared sample was vigorously mixed with a mechanical mixer for five min. Eventually, the external magnet was usage to gather the magnetic adsorbent (containing contaminant) to one side of the vial $^{21}$.

\section{c. Analyte desorption from the magnetic adsorbent}

To desorb analytes from the magnetic adsorbent, $5 \mathrm{~mL}$ of dichloromethane was poured and vortexed, and then the supernatant was thoroughly mixed with a whirlpool blender for three min. Next the sorbent was collected with magnets (exterior) on the sides of the vial. Previous step was conducted twaice, and afterward the sample was exposed to a mild flow of pure nitrogen gas in order to evaporate the solvent at $25^{\circ} \mathrm{C}$. The remainder was re-dissolved in acetonitrile /methanol (50:50 v/v, $50 \mu \mathrm{L}$ ) and the solution was vigorously shaken by the vortex-mixer (one minute). Eventually, one $\mu \mathrm{L}$ of the obtained solution was collected and injected with a syringe into the GC/MS. Additionally, optimization studies results demonstrated that the above-mentioned trend was permitted for recyclable extraction and quantitative analysis of polycyclic aromatic hydrocarbons from the samples ${ }^{8}$. Blank samples holding surrogate standard and control of quality samples were prepared and examined in the start, the middle, and eventually of each sample queue. Finally, all vegetable and fruit samples were tested in duplicate, and for quantification, their average values were utilized.

\subsection{Analytical and instrumental conditions}

The GC was Agilent 6890 (Agilent, PaloAlto, CA, America) with a detector of mass-selective in 5973 and the capillary column was DB-5 ms (30 m, 0.25 mm i.d., and $0.25 \mathrm{~mm}$ film thickness). In addition, instrumental temperatures included the temperature of the injector $290^{\circ} \mathrm{C}$ and the primary oven temperature of $70{ }^{\circ} \mathrm{C}$, that was held for one minute, raised to $300^{\circ} \mathrm{C}$ (rate of $10{ }^{\circ} \mathrm{C}$ min-1), and kept for seven minute. Further, the inlet functioned in the splitless mode, and temperature of the relocation line was kept at $300^{\circ} \mathrm{C}$. For gas of carrier, He (99.999\%) was utilized at a rate of $1 \mathrm{~mL} \mathrm{~min}^{-1}$ (constant flow). Furthermore, quadrupole, resource temperatures were maintained at 150 and $230{ }^{\circ} \mathrm{C}$, respectively, and the electron beam energy of the mass spectrometer was fixed at 70 $\mathrm{eV}$. The qualification was conducted based on the comparison between the acquired mass spectra and times of retention, and reference spectra and times of retentions. These times were acquired using injection calibration standards under identical GC-MS circumstances. Eventually, the analytes were measured by the GC/MS selected ion monitoring mode.

\subsection{Estimate of dietary exposure}


The risk of carcinogenic of a PAHs mixture is mainly represented using BaP equivalent level (BaPeq) and the toxicity equivalency factor (TEF) in Table 1, which is considered as a superior set for evaluating the potency of carcinogenic of PAH mixtures. Therefore, this set of TEFs was adopted to calculate BaPeq ${ }^{5}$ in the current study. The BaPeq of food (BEC) was conducted base to Eq (1).

Table1: PAHs and their toxic equivalent factors (TEFs)

\begin{tabular}{|llll|}
\hline PAHs & TEF & PAHs & TEF \\
\hline Benzo(a)pyrene (BaP) & 1 & Anthracene (ANT) & 0.01 \\
\hline Dibenz(a,h)anthracene (DahA) & 1 & Naphthalene (NAP) & 0.001 \\
\hline Benzo(k)fluoranthene (BkF) & 0.1 & Acenaphthylene (ACY) & 0.001 \\
\hline Indeno(1,2,3-cd)pyrene (IcdP) & 0.1 & Acenaphthene (ACE) & 0.001 \\
\hline Benz(a)anthracene (BaA) & 0.1 & Phenanthrene (PHE) & 0.001 \\
\hline Benzo(b)fluoranthene (BbF) & 0.1 & Fluorine (FLO) & 0.001 \\
\hline Chrysene (CHR) & 0.01 & Pyrene (PYR) & 0.001 \\
\hline Benzo(g,h,i)perylene (BghiP) & 0.01 & Fluoranthene (FLA) & 0.001 \\
\hline
\end{tabular}

$B E C=\sum_{i=1}^{n} C_{i} \times T E F$

where $\mathrm{Ci}$ and TEFi denote the level of the PAH congener $\mathrm{i}$ in vegetables and the TEF of the PAH congener i, respectively. For a singular PAH, the value is presumed to be $1 / 2$ of the respective LOD when the measured concentration is below the LOD. The carcinogenic potencies of these 16 PAHs were evaluated as the sum of each singular BaPeq.

Daily dietary PAH contact levels (ED) for each group were conducted by Eq (2).

$$
E_{D}=\sum_{i=1}^{n} B E C_{i} \times I R_{i}
$$

where BECi and IRj represent the BaPeq level of PAHs in food $i(\mathrm{ng} / \mathrm{g})$ and the amount of digestion of food $i$ per day $(\mathrm{g} / \mathrm{d})$, respectively ${ }^{20}$. Moreover, the amount of digestion of food by each group was gotten from questionnaires which were distributed among the citizens of Tehran.

\subsection{Evaluation of cancer risk}

The ILCR of individual groups in Tehran due to PAH dietary exposure was conducted according to Eq. (3).

$$
I L C R=E D \times E F \times E_{D} \times C S F \times C F / B W \times A T
$$

where ILCR is the incremental lifetime cancer risk of dietary exposure (dimensionless) and CSF indicates the oral cancer slope factor of BaP (7.3 per mg/kg/d) 22. In addition, $E_{D}, E D$, and BW denote the daily dietary PAH contact level (ng/d), exposure duration (year), and weight of body (70 kg), respectively. Finally, $A T$, $E F$, and $C F$ represent the main lifespan for carcinogens $(25,550 \text { days })^{23}$, the frequency of exposure (365 days/year), and the factor of conversion ( $10^{-6}$ $\mathrm{mg} / \mathrm{ng})$, respectively.

\subsection{Data analysis}

The risk assessment procedure is associated with uncertainty which may occur due to uncertainty in the measurement of factors. Therefore, uncertainty analysis is essential to achieving a more accurate result. In the current study, the uncertainty analysis of Monte Carlo was used to assess uncertainty in the exposure assessment. Further, the results were revealed as mean \pm SD, and the statistical analysis was performed by SPSS software, version 24.0 . Eventually, $1 / 2$ of the LOD was used to calculate the mean level in cases that PAH analytes were undetectable. For a better understanding of distribution of 16 PAHs among the vegetables and fruits samples marketed Tehran. Multivariate techniques were applied to evaluate the correlation between the type and amount of 16 PAHs and vegetables and fruits samples ${ }^{24}$. The PCA and HCA was conducted by the SPSS software (Version 18.0; Illinois, USA).

\section{Results And Discussion}

\subsection{Analytical method evaluation}

The analytical technique evaluated included the liquid extraction for PAHs and the SPE method by a magnetic nanoparticle sized composite (the first and second phases respectively). The extracted PAHs were investigated using GC-MS method. For the purpose of identification, a wide range of scan mass spectrums, four characteristic ion ratios, and the RTT of $\pm 0.5 \%$ tolerance criteria were applied for the quantification goal compared to the standard, followed by using the most intense ions from each compound. Next, these analytes were quantified by using the elected ion monitoring mode. Furthermore, the dwell time was determined at 100 min for each ion, followed by selecting GC conditions for reducing the test time and allowing all PAH compounds to elute in 
acquisition collections such as the ion number of appropriate for monitoring. According to Moazzen et al. (2013), one quantitation and two qualifier ions were controlled for each ingredient. Additionally, the conditions of optimum for the analysis were used for establishing the curves of calibration (0.050-

$150.000 \mu \mathrm{g} / \mathrm{kg}$ ) considering the correlation coefficient of 0.986-0.997. Then, the LOQ for each compound was determined based on the guideline of the council of international for harmonization ${ }^{8}$. Based on the results, the LOQs and limit of detection of PAH compounds were $0.105-0.240$ and $0.035-$ $0.080 \mu \mathrm{g} / \mathrm{kg}$, respectively. In addition, the method accuracy was assessed according to interday precision by the quality control analysis for the prepared samples at four concentrations on three repeated days. Further, the values of interday precision for all PAH compounds were less than 9.8, and the recorded values were $4.3-12.1$ and $6.1-20.3 \%$ for repeatability and reproducibility with an estimated recovery of $94.4-103.4 \%$, respectively. Furthermore, the feasibility and reliability of this method were confirmed by measuring PAHs in fruits and vegetables, and no interfering peak was observed in the internal standard area and analytes.

\subsection{The PAHs levels in fruits and vegetables}

Sixteen importance PAHs are introduced by the USEPA owing to their frequency in samples of food. BaP has been broadly introduced as a marker of PAHs carcinogenic in a limited number of foodstuffs. The European Commission first considered the BaP maximum level in various foodstuffs $(1.0 \mu \mathrm{g} / \mathrm{kg}$ for the processed cereal-based to $6.0 \mu \mathrm{g} / \mathrm{kg}$ for seafood). The concentration of measured $16 \mathrm{PAHs}$ is presented in Tables 2 and 3 . In the present study, BbF, BaP, BkF, DhA, BhP, and ICP were not detected in the fruit and vegetable samples, which is in agreement with the results of a study investigated in India 25 . 
Table 2

The mean of concentration of PAHs in fruits samples $(\mu \mathrm{g} / \mathrm{kg})$

\begin{tabular}{|c|c|c|c|c|c|c|c|c|c|c|c|c|c|c|c|c|c|}
\hline & & \multicolumn{16}{|c|}{ PAHs(ppb) } \\
\hline & & NAP & ACY & ACE & FLO & PHE & ANT & FLA & PYR & $\mathrm{BaA}$ & CHR & $\mathrm{BbF}$ & BkF & $\mathrm{BaP}$ & DahA & BghiP & IcdP \\
\hline \multirow[t]{5}{*}{ Citrus } & $\begin{array}{l}\text { Sweet } \\
\text { Lemon }\end{array}$ & $\begin{array}{l}60,4 \\
\pm 3,5\end{array}$ & $\begin{array}{l}19.5 \\
\pm 1.5\end{array}$ & $\begin{array}{l}38.1 \\
\pm 2.4\end{array}$ & $\begin{array}{l}7.9 \\
\pm 0,5\end{array}$ & $\begin{array}{l}13.1 \\
\pm 1,1\end{array}$ & $\begin{array}{l}15.8 \\
\pm 1.2\end{array}$ & $\begin{array}{l}5.9 \\
\pm 0,5\end{array}$ & $\begin{array}{l}6.1 \\
\pm 0,5\end{array}$ & n.d & $\begin{array}{l}2.7 \\
\pm 0,2\end{array}$ & n.d & n.d & n.d & n.d & n.d & n.d \\
\hline & Orange & $\begin{array}{l}94,1 \\
\pm 4,6\end{array}$ & $\begin{array}{l}24,1 \\
\pm 0,6\end{array}$ & $\begin{array}{l}28,7 \\
\pm 1,2\end{array}$ & $\begin{array}{l}12,9 \\
\pm 1,4\end{array}$ & $\begin{array}{l}7,8 \\
\pm 0,2\end{array}$ & $\begin{array}{l}11.4 \\
\pm 1.0\end{array}$ & $\begin{array}{l}10.9 \\
\pm 1.5\end{array}$ & $\begin{array}{l}7,1 \\
\pm 0.7\end{array}$ & $\begin{array}{l}1,2 \\
\pm \\
0,1\end{array}$ & n.d & n.d & n.d & n.d & n.d & n.d & n.d \\
\hline & Tangerine & $\begin{array}{l}70,4 \\
\pm 3,5\end{array}$ & $\begin{array}{l}36.7 \\
\pm 1.0\end{array}$ & $\begin{array}{l}32.7 \\
\pm 3.3\end{array}$ & $\begin{array}{l}19.3 \\
\pm 2.0\end{array}$ & $\begin{array}{l}11.9 \\
\pm 1,1\end{array}$ & $\begin{array}{l}9.2 \\
\pm \\
1 ., 2\end{array}$ & $\begin{array}{l}15.2 \\
\pm 1.3\end{array}$ & $\begin{array}{l}3.3 \\
\pm 0.3\end{array}$ & $\begin{array}{l}2.4 \\
\pm \\
0,2\end{array}$ & $\begin{array}{l}7.6 \\
\pm 0,1\end{array}$ & n.d & n.d & n.d & n.d & n.d & n.d \\
\hline & Grapefruit & $\begin{array}{l}106.4 \\
\pm 5.4\end{array}$ & $\begin{array}{l}5.4 \\
\pm 0,2\end{array}$ & $\begin{array}{l}35,3 \\
\pm 1,9\end{array}$ & $\begin{array}{l}42.2 \\
\pm 3,4\end{array}$ & $\begin{array}{l}2.4 \\
\pm 0,5\end{array}$ & $\begin{array}{l}3,3 \\
\pm 0,3\end{array}$ & $\begin{array}{l}11.2 \\
\pm 1.5\end{array}$ & $\begin{array}{l}5.2 \\
\pm 0.1\end{array}$ & $\begin{array}{l}0,1 \\
\pm \\
0.0\end{array}$ & $\begin{array}{l}4,1 \\
\pm 0,1\end{array}$ & n.d & n.d & n.d & n.d & n.d & n.d \\
\hline & Lemon & $\begin{array}{l}97,9 \\
\pm 4,2\end{array}$ & $\begin{array}{l}21.7 \\
\pm 2.0\end{array}$ & $\begin{array}{l}32.0 \\
\pm 2 \\
6\end{array}$ & $\begin{array}{l}10.8 \\
\pm 1.1\end{array}$ & $\begin{array}{l}5.1 \\
\pm 0.5\end{array}$ & $\begin{array}{l}20.6 \\
\pm 1.0\end{array}$ & $\begin{array}{l}11.0 \\
\pm 1.4\end{array}$ & $\begin{array}{l}3.0 \\
\pm 0.4\end{array}$ & n.d & n.d & n.d & n.d & n.d & n.d & n.d & n.d \\
\hline \multirow[t]{9}{*}{$\begin{array}{l}\text { Other } \\
\text { fruits }\end{array}$} & Apple & $\begin{array}{l}56.0 \\
\pm 4.4\end{array}$ & $\begin{array}{l}19,0 \\
\pm 1.1\end{array}$ & $\begin{array}{l}28.4 \\
\pm 2.4\end{array}$ & $\begin{array}{l}11,0 \\
\pm 1.0\end{array}$ & $\begin{array}{l}5.7 \\
\pm 0.6\end{array}$ & $\begin{array}{l}14.9 \\
\pm 1.6\end{array}$ & $\begin{array}{l}11,0 \\
\pm \\
0.16\end{array}$ & $\begin{array}{l}4.7 \\
\pm 0.7\end{array}$ & $\begin{array}{l}3.5 \\
\pm \\
0.5\end{array}$ & n.d & n.d & n.d & n.d & n.d & n.d & n.d \\
\hline & Banana & $\begin{array}{l}70.6 \\
\pm 3.5\end{array}$ & $\begin{array}{l}10.1 \\
\pm 1.1\end{array}$ & $\begin{array}{l}44.5 \\
\pm \\
2.56\end{array}$ & $\begin{array}{l}5.8 \\
\pm 0.2\end{array}$ & $\begin{array}{l}16.8 \\
\pm 1.1\end{array}$ & $\begin{array}{l}24.6 \\
\pm 3.2\end{array}$ & $\begin{array}{l}3.7 \\
\pm 0.4\end{array}$ & $\begin{array}{l}9,0 \\
\pm 1,0\end{array}$ & n.d & $\begin{array}{l}5.4 \\
\pm 0.1\end{array}$ & n.d & n.d & n.d & n.d & n.d & n.d \\
\hline & Kiwi & $\begin{array}{l}106.1 \\
\pm 6.0\end{array}$ & $\begin{array}{l}15.8 \\
\pm 1 \\
9\end{array}$ & $\begin{array}{l}25.2 \\
\pm 1.8\end{array}$ & $\begin{array}{l}16.6 \\
\pm 0.8\end{array}$ & $\begin{array}{l}12.6 \\
\pm 0.5\end{array}$ & $\begin{array}{l}6.3 \\
\pm 0 \\
8\end{array}$ & $\begin{array}{l}6.5 \\
\pm 0.8\end{array}$ & $\begin{array}{l}5.8 \\
\pm 0.1\end{array}$ & n.d & n.d & n.d & n.d & n.d & n.d & n.d & n.d \\
\hline & Pomegranate & $\begin{array}{l}81.4 \\
\pm 4.8\end{array}$ & $\begin{array}{l}6.7 \\
\pm 1.1\end{array}$ & $\begin{array}{l}27.4 \\
\pm 2.1\end{array}$ & $\begin{array}{l}11.0 \\
\pm 1.7\end{array}$ & $\begin{array}{l}5.2 \\
\pm 0.2\end{array}$ & $\begin{array}{l}5.3 \\
\pm 0 \\
8\end{array}$ & $\begin{array}{l}4.3 \\
\pm 0.8\end{array}$ & $\begin{array}{l}4.29 \\
\pm 0.1\end{array}$ & $\begin{array}{l}1.4 \\
\pm \\
0.1\end{array}$ & $\begin{array}{l}2.4 \\
\pm 0.0\end{array}$ & n.d & n.d & n.d & n.d & n.d & n.d \\
\hline & Peach & $\begin{array}{l}73.3 \\
\pm 4.7\end{array}$ & $\begin{array}{l}18.0 \\
\pm 1.1\end{array}$ & $\begin{array}{l}20.2 \\
\pm 1.4\end{array}$ & $\begin{array}{l}11.1 \\
\pm 1.0\end{array}$ & $\begin{array}{l}3.4 \\
\pm 0.3\end{array}$ & $\begin{array}{l}8.9 \\
\pm 0.6\end{array}$ & $\begin{array}{l}7.9 \\
\pm 0.1\end{array}$ & $\begin{array}{l}8.5 \\
\pm 0.2\end{array}$ & $\begin{array}{l}2.4 \\
\pm \\
0.1\end{array}$ & $\begin{array}{l}3.2 \\
\pm 0.1\end{array}$ & n.d & n.d & n.d & n.d & n.d & n.d \\
\hline & Nectarine & $\begin{array}{l}103.2 \\
\pm 5.2\end{array}$ & $\begin{array}{l}30.6 \\
\pm 4.5\end{array}$ & $\begin{array}{l}38.5 \\
\pm 2.1\end{array}$ & $\begin{array}{l}14.9 \\
\pm 1.5\end{array}$ & $\begin{array}{l}8.4 \\
\pm 0.5\end{array}$ & $\begin{array}{l}14.3 \\
\pm 2.0\end{array}$ & $\begin{array}{l}5.2 \\
\pm 2.4\end{array}$ & $\begin{array}{l}6.2 \\
\pm 0.2\end{array}$ & $\begin{array}{l}1.3 \\
\pm 0 \\
1\end{array}$ & $\begin{array}{l}2.1 \\
\pm 0.1\end{array}$ & n.d & n.d & n.d & n.d & n.d & n.d \\
\hline & Plums & $\begin{array}{l}56.3 \\
\pm 3.2\end{array}$ & $\begin{array}{l}30.7 \\
\pm 3.1\end{array}$ & $\begin{array}{l}33.6 \\
\pm 1.1\end{array}$ & $\begin{array}{l}16.3 \\
\pm 1.1\end{array}$ & $\begin{array}{l}3.6 \\
\pm 0.7\end{array}$ & $\begin{array}{l}29 . \\
9 \pm \\
2.0\end{array}$ & $\begin{array}{l}17.5 \\
\pm 1.5\end{array}$ & $\begin{array}{l}7.3 \\
\pm 0.1\end{array}$ & $\begin{array}{l}1.2 \\
\pm \\
0.1\end{array}$ & $\begin{array}{l}12.8 \\
\pm 1.2\end{array}$ & n.d & n.d & n.d & n.d & n.d & n.d \\
\hline & Persimmon & $\begin{array}{l}67.7 \\
\pm 3.2\end{array}$ & $\begin{array}{l}9.5 \\
\pm 0.2\end{array}$ & $\begin{array}{l}23.4 \\
\pm 2.6\end{array}$ & $\begin{array}{l}11.1 \\
\pm 1.1\end{array}$ & $\begin{array}{l}1.5 \\
\pm 0.1\end{array}$ & $\begin{array}{l}18.5 \\
\pm 2.1\end{array}$ & $\begin{array}{l}6.3 \\
\pm 0.2\end{array}$ & $\begin{array}{l}2.3 \\
\pm 0.2\end{array}$ & n.d & n.d & n.d & n.d & n.d & n.d & n.d & n.d \\
\hline & Grapes & $\begin{array}{l}107,0 \\
\pm 6,0\end{array}$ & $\begin{array}{l}6.4 \\
\pm 0.1\end{array}$ & $\begin{array}{l}35.4 \\
\pm 2.2\end{array}$ & $\begin{array}{l}47.7 \\
\pm 1.1\end{array}$ & $\begin{array}{l}2.2 \\
\pm 0.3\end{array}$ & $\begin{array}{l}18.8 \\
\pm 2.7\end{array}$ & $\begin{array}{l}9.0 \\
\pm 0.0\end{array}$ & $\begin{array}{l}11.6 \\
\pm 2.1\end{array}$ & n.d & $\begin{array}{l}14.3 \\
\pm 2.9\end{array}$ & n.d & n.d & n.d & n.d & n.d & n.d \\
\hline \multirow[t]{4}{*}{ Melons } & Watermelon & $\begin{array}{l}68.9 \\
\pm 4.2\end{array}$ & $\begin{array}{l}13.6 \\
\pm 1.2\end{array}$ & $\begin{array}{l}27.2 \\
\pm 3.9\end{array}$ & $\begin{array}{l}6.5 \\
\pm 0,2\end{array}$ & $\begin{array}{l}1.9 \\
\pm 0,2\end{array}$ & $\begin{array}{l}17.0 \\
\pm 3.8\end{array}$ & $\begin{array}{l}17.5 \\
\pm 3.1\end{array}$ & $\begin{array}{l}2.3 \\
\pm 0.1\end{array}$ & $\begin{array}{l}0.1 \\
\pm \\
0.0\end{array}$ & n.d & n.d & n.d & n.d & n.d & n.d & n.d \\
\hline & Cantaloupe & $\begin{array}{l}43,0 \\
\pm 3.0\end{array}$ & $\begin{array}{l}17.4 \\
\pm 1.3\end{array}$ & $\begin{array}{l}25.2 \\
\pm 3.0\end{array}$ & $\begin{array}{l}6.6 \\
\pm 0.1\end{array}$ & $\begin{array}{l}1,0 \\
\pm 0.1\end{array}$ & $\begin{array}{l}20.7 \\
\pm 3.8\end{array}$ & $\begin{array}{l}7.2 \\
\pm 0.3\end{array}$ & $\begin{array}{l}2.3 \\
\pm 0.1\end{array}$ & n.d & n.d & n.d & n.d & n.d & n.d & n.d & n.d \\
\hline & Melon & $\begin{array}{l}61.0 \\
\pm 3.9\end{array}$ & $\begin{array}{l}24,0 \\
\pm 3.0\end{array}$ & $\begin{array}{l}47.1 \\
\pm 6.6\end{array}$ & $\begin{array}{l}36.2 \\
\pm 4.5\end{array}$ & $\begin{array}{l}4.3 \\
\pm 0.0\end{array}$ & $\begin{array}{l}15.2 \\
\pm 1.7\end{array}$ & $\begin{array}{l}14.2 \\
\pm 2.2\end{array}$ & $\begin{array}{l}8.0 \\
\pm 0.1\end{array}$ & n.d & n.d & n.d & n.d & n.d & n.d & n.d & n.d \\
\hline & Cucumber & $\begin{array}{l}95.9 \\
\pm 3.9\end{array}$ & $\begin{array}{l}24,0 \\
\pm \\
1.90\end{array}$ & $\begin{array}{l}41.7 \\
\pm 5.3\end{array}$ & $\begin{array}{l}12.3 \\
\pm 1.0\end{array}$ & $\begin{array}{l}10.8 \\
\pm 1.1\end{array}$ & $\begin{array}{l}25.6 \\
\pm 5.2\end{array}$ & $\begin{array}{l}9.9 \\
\pm 1.0\end{array}$ & $\begin{array}{l}5,0 \\
\pm 0.1\end{array}$ & n.d & n.d & n.d & n.d & n.d & n.d & n.d & n.d \\
\hline
\end{tabular}


Table 3

The mean of concentration of PAHs in vegetables samples

\begin{tabular}{|c|c|c|c|c|c|c|c|c|c|c|c|c|c|c|c|c|}
\hline & & \multicolumn{15}{|c|}{ PAHs ( $\mu g / k g)$} \\
\hline & & NAP & ACY & ACE & FLO & PHE & ANT & FLA & PYR & $\mathrm{BaA}$ & CHR & $\mathrm{BbF}$ & BkF & $\mathrm{BaP}$ & DahA & BghiP \\
\hline \multirow[t]{6}{*}{$\begin{array}{l}\text { Root } \\
\text { Vegetables }\end{array}$} & Beet & $\begin{array}{l}48.4 \\
\pm 3.5\end{array}$ & $\begin{array}{l}3.9 \pm \\
0.2\end{array}$ & $\begin{array}{l}24.5 \pm \\
3.3\end{array}$ & $\begin{array}{l}6.8 \\
\pm 0.9\end{array}$ & $\begin{array}{l}9.4 \\
\pm 0.4\end{array}$ & $\begin{array}{l}11.6 \\
\pm 1.3\end{array}$ & $\begin{array}{l}5.3 \\
\pm 0.9\end{array}$ & $\begin{array}{l}9.1 \\
\pm 1.0\end{array}$ & n.d & $\begin{array}{l}0.5 \\
\pm 0.4\end{array}$ & n.d & n.d & n.d & n.d & n.d \\
\hline & Carrot & $\begin{array}{l}72.1 \\
\pm 2.3\end{array}$ & $\begin{array}{l}13.3 \\
\pm 1.4\end{array}$ & $\begin{array}{l}17.7 \pm \\
2.0\end{array}$ & $\begin{array}{l}6.7 \\
\pm 0.9\end{array}$ & $\begin{array}{l}9.4 \\
\pm 1.2\end{array}$ & $\begin{array}{l}11.3 \\
\pm 1.0\end{array}$ & $\begin{array}{l}8.4 \\
\pm 0.8\end{array}$ & $\begin{array}{l}7.5 \\
\pm 1.3\end{array}$ & $\begin{array}{l}2,0 \\
\pm \\
1.1\end{array}$ & n.d & n.d & n.d & n.d & n.d & n.d \\
\hline & Potato & $\begin{array}{l}114.1 \\
\pm 5.0\end{array}$ & $\begin{array}{l}19.5 \\
\pm 2.0\end{array}$ & $\begin{array}{l}50.5 \pm \\
6.4\end{array}$ & $\begin{array}{l}154 \\
\pm 1.8\end{array}$ & $\begin{array}{l}12.9 \\
\pm 2.4\end{array}$ & $\begin{array}{l}10.4 \\
\pm 1.9\end{array}$ & $\begin{array}{l}9.66 \\
\pm 1.3\end{array}$ & $\begin{array}{l}4.64 \\
\pm 1.0\end{array}$ & n.d & $\begin{array}{l}4.8 \\
\pm 0.0\end{array}$ & n.d & n.d & n.d & n.d & n.d \\
\hline & Turnip & $\begin{array}{l}79.0 \\
\pm 3.4\end{array}$ & $\begin{array}{l}16.2 \\
\pm 1.0\end{array}$ & $\begin{array}{l}33.53 \\
\pm 5.0\end{array}$ & $\begin{array}{l}11,8 \\
\pm 1.5\end{array}$ & $\begin{array}{l}5.7 \\
\pm 1.0\end{array}$ & $\begin{array}{l}12.6 \\
\pm 1.9\end{array}$ & $\begin{array}{l}6.5 \\
\pm 0.9\end{array}$ & $\begin{array}{l}5.4 \\
\pm 0.8\end{array}$ & n.d & $\begin{array}{l}3.1 \\
\pm 1.0\end{array}$ & n.d & n.d & n.d & n.d & n.d \\
\hline & Onion & $\begin{array}{l}79.2 \\
\pm 4.4\end{array}$ & $\begin{array}{l}16.6 \\
\pm 1.0\end{array}$ & $\begin{array}{l}31.5 \pm \\
3.1\end{array}$ & $\begin{array}{l}13.6 \\
\pm 2.1\end{array}$ & $\begin{array}{l}5.4 \\
\pm 0.9\end{array}$ & $\begin{array}{l}7.8 \\
\pm 1.5\end{array}$ & $\begin{array}{l}7.4 \\
\pm 1.3\end{array}$ & $\begin{array}{l}3.1 \\
\pm 0.0\end{array}$ & $\begin{array}{l}1.4 \\
\pm \\
0.6\end{array}$ & $\begin{array}{l}11.2 \\
\pm 1.0\end{array}$ & n.d & n.d & n.d & n.d & n.d \\
\hline & Radish & $\begin{array}{l}66.6 \\
\pm 3.0\end{array}$ & $\begin{array}{l}9.4 \pm \\
1.1\end{array}$ & $\begin{array}{l}26.8 \pm \\
4.1\end{array}$ & $\begin{array}{l}15.3 \\
\pm 2.4\end{array}$ & $\begin{array}{l}9.5 \\
\pm 1.1\end{array}$ & $\begin{array}{l}11.5 \\
\pm 1.0\end{array}$ & $\begin{array}{l}8.3 \\
\pm 1.1\end{array}$ & $\begin{array}{l}6.5 \\
\pm 1.0\end{array}$ & $\begin{array}{l}1.1 \\
\pm \\
0.2\end{array}$ & $\begin{array}{l}2.33 \\
\pm 0.8\end{array}$ & n.d & n.d & n.d & n.d & n.d \\
\hline \multirow[t]{3}{*}{ Cabbages } & $\begin{array}{l}\text { White } \\
\text { cabbage }\end{array}$ & $\begin{array}{l}108.2 \\
\pm 6.1\end{array}$ & $\begin{array}{l}17.5 \\
\pm 1.1\end{array}$ & $\begin{array}{l}29.5 \pm \\
2.0\end{array}$ & $\begin{array}{l}13.8 \\
\pm 1.1\end{array}$ & $\begin{array}{l}8.6 \\
\pm 0.9\end{array}$ & $\begin{array}{l}14.5 \\
\pm 2,0\end{array}$ & $\begin{array}{l}8.2 \\
\pm 0.1\end{array}$ & $\begin{array}{l}4.8 \\
\pm 0.5\end{array}$ & n.d & $\begin{array}{l}2.6 \\
\pm 0.1\end{array}$ & n.d & n.d & n.d & n.d & n.d \\
\hline & $\begin{array}{l}\text { Purple } \\
\text { cabbage }\end{array}$ & $\begin{array}{l}102.4 \\
\pm 5.4\end{array}$ & $\begin{array}{l}5.1 \pm \\
0.4\end{array}$ & $\begin{array}{l}26.8 \pm \\
3.1\end{array}$ & $\begin{array}{l}35.6 \\
\pm 3.1\end{array}$ & nd & n.d & $\begin{array}{l}83 \pm \\
1.1\end{array}$ & $\begin{array}{l}16.3 \\
\pm 1.1\end{array}$ & n.d & $\begin{array}{l}11.3 \\
\pm 1.1\end{array}$ & n.d & n.d & n.d & n.d & n.d \\
\hline & Cauliflower & $\begin{array}{l}43.1 \\
\pm 2.1\end{array}$ & $\begin{array}{l}28.65 \\
\pm 1.9\end{array}$ & $\begin{array}{l}30.6 \pm \\
4.1\end{array}$ & $\begin{array}{l}19.9 \\
\pm 2.1\end{array}$ & $\begin{array}{l}4.5 \\
\pm 0.8\end{array}$ & $\begin{array}{l}6.7 \\
\pm 1.7\end{array}$ & $\begin{array}{l}12.6 \\
\pm 1.2\end{array}$ & $\begin{array}{l}4.5 \\
\pm 0.3\end{array}$ & n.d & n.d & n.d & n.d & n.d & n.d & n.d \\
\hline \multirow[t]{3}{*}{$\begin{array}{l}\text { leafy } \\
\text { vegetables }\end{array}$} & Lettuce & $\begin{array}{l}63.2 \\
\pm 5.0\end{array}$ & $\begin{array}{l}0.4 \pm \\
1.0\end{array}$ & $\begin{array}{l}44.7 \pm \\
4.1\end{array}$ & $\begin{array}{l}9.3 \\
\pm 0.7\end{array}$ & $\begin{array}{l}19.1 \\
\pm 3.1\end{array}$ & $\begin{array}{l}20.9 \\
\pm 2.0\end{array}$ & $\begin{array}{l}3.9 \\
\pm 0.4\end{array}$ & $\begin{array}{l}5.3 \\
\pm 0.5\end{array}$ & n.d & $\begin{array}{l}3.6 \\
\pm 0.1\end{array}$ & n.d & n.d & n.d & n.d & n.d \\
\hline & Spinach & $\begin{array}{l}46.5 \\
\pm 4.0\end{array}$ & $\begin{array}{l}10.3 \\
\pm 1.0\end{array}$ & $\begin{array}{l}22.6 \pm \\
2.1\end{array}$ & $\begin{array}{l}9.4 \\
\pm 1.1\end{array}$ & n.d & n.d & $\begin{array}{l}5.6 \\
\pm 0.1\end{array}$ & $\begin{array}{l}10.3 \\
\pm 1.1\end{array}$ & n.d & n.d & n.d & n.d & n.d & n.d & n.d \\
\hline & $\begin{array}{l}\text { Mixture of } \\
\text { Leek, } \\
\text { Coriander } \\
\text { and Basil }\end{array}$ & $\begin{array}{l}104.8 \\
\pm 6.5\end{array}$ & $\begin{array}{l}19.2 \\
\pm 2.3\end{array}$ & $\begin{array}{l}135.10 \\
\pm 7.10\end{array}$ & $\begin{array}{l}10.9 \\
\pm 1.1\end{array}$ & $\begin{array}{l}2.4 \\
\pm 0.5\end{array}$ & $\begin{array}{l}24.9 \\
\pm 3.1\end{array}$ & $\begin{array}{l}13.2 \\
\pm 1.1\end{array}$ & $\begin{array}{l}4.3 \\
\pm 0.6\end{array}$ & nd & n.d & n.d & n.d & n.d & n.d & n.d \\
\hline \multirow[t]{2}{*}{$\begin{array}{l}\text { Fruit } \\
\text { Vegetable }\end{array}$} & Eggplant & $\begin{array}{l}84.5 \\
\pm 4.7\end{array}$ & $\begin{array}{l}12.1 \\
\pm 1.0\end{array}$ & $\begin{array}{l}24.9 \pm \\
2.12\end{array}$ & $\begin{array}{l}7.2 \\
\pm 0.1\end{array}$ & $\begin{array}{l}10.6 \\
\pm 1.4\end{array}$ & $\begin{array}{l}19.4 \\
\pm 1.1\end{array}$ & $\begin{array}{l}12.2 \\
\pm 1.1\end{array}$ & $\begin{array}{l}4.4 \\
\pm 1.1\end{array}$ & n.d & n.d & n.d & n.d & n.d & n.d & n.d \\
\hline & Tomato & $\begin{array}{l}109.2 \\
\pm 6.3\end{array}$ & $\begin{array}{l}7.4 \pm \\
0.8\end{array}$ & $\begin{array}{l}20.4 \pm \\
2.0\end{array}$ & $\begin{array}{l}23.5 \\
\pm 1.9\end{array}$ & n.d & $\begin{array}{l}5.4 \\
\pm 0.1\end{array}$ & $\begin{array}{l}18.8 \\
\pm 1.4\end{array}$ & $\begin{array}{l}6.12 \\
\pm 0.1\end{array}$ & $\begin{array}{l}2.3 \\
\pm \\
0.1\end{array}$ & $\begin{array}{l}4.3 \\
\pm 0.1\end{array}$ & n.d & n.d & n.d & n.d & n.d \\
\hline
\end{tabular}

Some studies revealed that fruits and vegetables can be contaminated from water, soil, and air (e.g., wastes burning, industries, and urban activities) ${ }^{26,27}$.

Table 2, 3 show the mean concentration of the NAP $(42.9-114.1 \mu \mathrm{g} / \mathrm{kg})$ and ACE $(17.7-135.1 \mu \mathrm{g} / \mathrm{kg})$ in all vegetable and fruit samples had the highest level compared to other PAHs.

In addition, the obtained data approved that NAP, ACY, FLO, PHE, ANT, FLA, and PYR were analyzed in entire samples. However, CHR was not found in fruit samples such as orange, lemon, apple, kiwi, nectarine, persimmon, grape, cantaloupes, and cucumber. Also, BaA was not observed in sweet lemon, lemon, banana, kiwi, peach, nectarine, persimmon, watermelon, and cucumber. Conversely, the concentration of BaA in vegetable samples was found in the collected carrot, onion, radish, and the mixture of leek, coriander, and basil samples and, CHR was observed in the beet, potato, turnip, onion, radish, tomato, and white and purple cabbage. The results also revealed that PHE and ANT were not found in purple cabbage, spinach, tomato samples, and in purple cabbage and spinach samples, respectively.

The means of the PAHs based on fruit type are shown in Table 2. The results showed that the sum of 16 PAHs in the fruits, ranged from 123.2 to $252.4 \mu \mathrm{g} / \mathrm{kg}$, and the highest and lowest ones were detected in cucumber and persimmon, respectively. Further, the sum of 8 PAHs in the fruits varied in the range n.d. $-14.3 \mu \mathrm{g} / \mathrm{kg}$, and total rank of PAHs in the three groups of fruits was citrus $>$ other fruits $>$ melons.

The data related to the means of PAHs accordance with the type of vegetables are revealed in Table 3. results proved that the sum of 16 PAHs in vegetables was in $104.7-314.9 \mu \mathrm{g} / \mathrm{kg}$ and the highest and lowest ones were found in white cabbage and spinach, respectively. Eventually, the sum of 8 PAHs in vegetables varied in the range n.d. to $12.6 \mu \mathrm{g} / \mathrm{kg}$, and the total rank of PAHs in the four groups of vegetables was leafy vegetables >cabbage > fruit vegetables $>$ root vegetables. 
In addition, it was found that the fruits and vegetables studied in the present study have a relatively higher concentration of 16 PAH than other countries, which may be due to soil and air pollution.

In another study, Camargo and Toledo indicated that the mean level of lettuce was $17.9 \mu \mathrm{g} / \mathrm{kg}$, which was less than the measured amount in the current study. Additionally, the total PAH content was $4.1,3.9$, and3.8 $\mu \mathrm{g} / \mathrm{kg}$ in apple, grape, and pear, respectively ${ }^{28}$. Based on the evaluation of various food groups. Martorell et al. revealed that the total PAHs levels in fruits and vegetables were 810 and $1220 \mu \mathrm{g} / \mathrm{kg}$, respectively ${ }^{2}$. In addition, Lee et al. concluded that the mean level of four and eight PAHs were $0.2 \mu \mathrm{g} / \mathrm{kg}$, and $0.7 \mu \mathrm{g} / \mathrm{kg}$ in fruit samples, respectively ${ }^{29}$. According to the reports of Veyrand et al., the concentration of BaP was $0.01 \mu \mathrm{g} / \mathrm{kg}$, and that of the four PAHs was $0.04 \mu \mathrm{g} / \mathrm{kg}$ in vegetables. In another study, the level of BaP was 46.9-17and 55.5$343.4 \mu \mathrm{g} / \mathrm{kg}$ in carrot and potato, respectively ${ }^{30}$.

PAHs are distributed from air, soil, and water. Further, air pollution is regarded as a crucial source and route which can transfer contaminants such as PAHs to plants including different vegetables and fruits. ${ }^{31}$

The studies indicated that the PAHs can probably be absorbed on the suspended particle in the air and these particles can substantially deposit on the studied plants, leading to the transfer of PAHs from particles to the leaves cuticle. Furthermore, hydrophobic fruits and vegetables can directly adsorb PAHs from the particles ${ }^{6,32}$.

In another study, the PAHs analyzed in vegetables that were collected from near an industrial area ${ }^{33}$. However, the adsorption of volatile organic compounds probably is reinforced with increasing surface area because large surfaces have more exchange with the gas phase and can facilitate plant contamination. This is observed in the case of leaf vegetables which have a higher exchange rate than other vegetables and fruits. For example, in the samples obtained from near a chemical company, the total PAHs content was higher in leaves of cabbage and maize (with nearly $4.2 \pm 3.5$ and $2.4 \pm 1.8 \mu \mathrm{g} / \mathrm{kg}$ wet weight, respectively) compared to grape $\left(0.3 \pm 0.2 \mathrm{mg} / \mathrm{kg}\right.$ ) and tomato (about $0.09 \pm 0.04 \mathrm{mg} / \mathrm{kg}$ ) ${ }^{33}$. In the present study, the surveyed white cabbage samples had the maximum concentration of PAHs $(202 \mu \mathrm{g} / \mathrm{kg})$, that is steady with the results of previous research.

The another research was shown that the accumulation of contaminant is commonly extremely more in vegetables and fruits which have longer growing periods ${ }^{34}$, which corroborates the outcomes of the present research, representing which the concentrations of PAHs were higher in white cabbage (202 $\left.\mu \mathrm{g} / \mathrm{kg}\right)$ and a mixture of leek, coriander, and basil $(201 \mu \mathrm{g} / \mathrm{kg})$.

According to the results of a study performed in France, PAHs are probable to be more amassed in crops which are located in urban or industrial areas compared to those in rural ones. The trace concentration of compounds including PHE, FLA, and PYR are obtained in every raw vegetable and fruit, and relatively high amounts of lighter PAHs including NAP, ACY, and ACE are reported in some of fruit and vegetable ${ }^{33}$.

In the study by Ashraf and Salam, the total 8PAHs level in root vegetables such as potatoes and carrots revealed higher levels ( $11 \mu \mathrm{g} / \mathrm{kg})$, while turnip revealed moderately lower level at $9.3 \mu \mathrm{g} / \mathrm{kg}$. Also, the highest levels of BaP were found in potatoes and turnips $2.1 \pm 1.1 \mu \mathrm{g} / \mathrm{kg}$ and $2.1 \pm 1.1 \mu \mathrm{g} / \mathrm{kg}$, respectively ${ }^{35}$.

In the study of Abou Arab AAK et al. in some Egyptian vegetables and fruits, the highest concentration of total PAHs was observed in spinach, potato, apple and guava $9.0 \mu \mathrm{g} / \mathrm{kg} 6.2 \mu \mathrm{g} / \mathrm{kg}, 2.9 \mu \mathrm{g} / \mathrm{kg}$ and $2.3 \mu \mathrm{g} / \mathrm{kg}$, respectively ${ }^{26}$.

The occurrence of PAHs in products relies on the environment of the plants (e.g., soil, water, and air), the kind of plants, and growing time as well as the proximity of plants farms to industrial centers and high-traffic highways.

To reduce environmental pollution, the PAHs production cycle in the environment must be avoided. In addition, measures must be taken to reduce the PAHs content in agricultural water, air and soil. Therefore, it is necessary to study the sources of water, air and soil as well as start awareness campaigns about the carcinogenic effects, high consumption of these compounds through various foods and ways to prevent it.

\subsection{Chemometric Analysis}

PCA performed to the data sets corresponding to 16 PAHs between the vegetables and fruits samples. Four principal components extracted for $71.6 \%$ of the total variance (Fig. 1). FLO, CHR and PYR were the closest, indicating that is variables had similar behavior. The first factor (PC1) calculated for $25.03 \%$ of the variance and characterized using a spectrum of PAHs. This compound had a high positive correlation with NAP, ACE, FLO, CHR and Total PAH, but they had a significant and negative correlation with PHE. Meanwhile, the second factor (PC2) explained $21.09 \%$ of the total variance, and this component had high positive correlation with ANT, ACY and ACE, but they had a significant and negative correlation with PYR, CHR and FLO. The PC3 explicated $15 \%$ of the total variance, and this component had high positive correlation with BaP and ACE, but they had a significant and negative correlation with NAP, ACE and PHE.

HCA can be used to create connected clusters among the samples by distance of their attributes ${ }^{36}$. In current study, HCA was conducted to further interpret the levels of $16 \mathrm{PAHs}$ in vegetables and fruits samples using average linkage.

As shown in Fig. 2, the clustering results of 16 PAHs in vegetables and fruits samples were slightly different. In the total samples, resulting dendrogram showed two main groups composed. One cluster contains two sub-groups with all samples except mixture of leek, coriander, and basil samples, while the second cluster includes only mixture of leek, coriander, and basil samples was difference from other samples. In evaluate the correlation between the type and amount of 16 PAHs in vegetables and fruits samples, resulting dendrogram showed two groups composed. One cluster includes only PHE and ANT. This indicated that the distribution of PHE was more similar to that of ANT. The second cluster contains two sub-groups: one with BaA, FLA and ACY, while the second cluster includes other PAHs. 


\subsection{Daily exposure estimation of PAHs}

The daily exposure estimation of PAHs was conducted as presented in Sect. 2.5. Total consumption of vegetables and fruits per person per day was calculated through a food frequency questionnaire, followed by calculating dietary intake with the PAHs concentration in vegetables and fruits. According to data in Table 4, the highest level of daily dietary in four groups of vegetables was detected in fruits vegetables (3378.4 $\mu \mathrm{g} / \mathrm{kg} / \mathrm{day})$ and then root vegetables $(1251.5 \mu \mathrm{g} / \mathrm{kg} /$ day $)$. Also, the lowest concentration of daily dietary was found in radish $(0.6 \mu \mathrm{g} / \mathrm{kg} / \mathrm{day})$ and cauliflower $(2.88 \mu \mathrm{g} / \mathrm{kg} / \mathrm{day})$. Moreover, the highest level of daily dietary in three groups of fruits was related to melons $(4,069.8 \mu \mathrm{g} / \mathrm{kg} / \mathrm{day})$, other fruits $(1,315.7 \mu \mathrm{g} / \mathrm{kg} / \mathrm{day})$ and citrus $(101.0 \mu \mathrm{g} / \mathrm{kg} /$ day $)$, respectively.

Table 4

Uncertainty analysis for the EDi and ILCR of investigated PAH content in vegetables samples

\begin{tabular}{|c|c|c|c|c|c|c|c|}
\hline & & \multicolumn{3}{|c|}{ EDi $(\mu \mathrm{g} / \mathrm{kg} / \mathrm{d})$} & \multicolumn{3}{|l|}{ ILCRi } \\
\hline & & $50 \%$ Perc & 75\% Perc & $95 \%$ Perc & $50 \%$ Perc & 75\% Perc & $95 \%$ Perc \\
\hline \multirow[t]{6}{*}{ Root Vegetables } & Beet & 20.6 & 22.7 & 26.1 & $2.13 \mathrm{E}-06$ & $2.5 \mathrm{E}-06$ & $3.4 \mathrm{E}-06$ \\
\hline & Carrot & 37.7 & 40.9 & 47.2 & 4.01E-06 & $4.8 \mathrm{E}-06$ & $6.0 \mathrm{E}-06$ \\
\hline & Potato & 990.6 & $1,085.3$ & $1,300.5$ & $1.02 \mathrm{E}-04$ & $1.23 \mathrm{E}-04$ & $1.58 \mathrm{E}-04$ \\
\hline & Tumip & 7.6 & 8.3 & 9.5 & $7.8 \mathrm{E}-07$ & $9.34 \mathrm{E}-07$ & 1.19E-06 \\
\hline & Onion & 507.9 & 558.9 & 639.7 & $5.2 \mathrm{E}-05$ & $6.2 \mathrm{E}-05$ & 8.1E-05 \\
\hline & Radish & 0.4 & 0.5 & 0.6 & 4.7E-08 & $5.6 \mathrm{E}-08$ & 7.1E-08 \\
\hline \multirow[t]{3}{*}{ Cabbages } & White cabbage & 11.7 & 12.8 & 14.5 & $1.2 \mathrm{E}-06$ & $1.4 \mathrm{E}-06$ & $1.9 \mathrm{E}-06$ \\
\hline & Purple cabbage & 10.8 & 11.9 & 13.4 & $1.1 \mathrm{E}-06$ & $1.4 \mathrm{E}-06$ & 1.7E-06 \\
\hline & Cauliflower & 2.3 & 2.5 & 2.8 & $2.4 \mathrm{E}-07$ & $2.8 \mathrm{E}-07$ & 3.5E-07 \\
\hline \multirow[t]{3}{*}{ leafy vegetables } & Lettuce & 21.6 & 23.7 & 27.04 & $2.2 \mathrm{E}-06$ & $2.6 \mathrm{E}-06$ & 3. $5 \mathrm{E}-06$ \\
\hline & Spinach & 5.3 & 5.8 & 6.6 & $5.4 \mathrm{E}-07$ & 6. $5 \mathrm{E}-07$ & 8. $6 \mathrm{E}-07$ \\
\hline & Mixture of Leek, Coriander and Basil & 21.6 & 23.8 & 26.8 & $2.2 \mathrm{E}-06$ & $2.6 \mathrm{E}-06$ & 3.5E-06 \\
\hline \multirow[t]{2}{*}{ Fruit Vegetable } & Eggplant & 231.1 & 255.3 & 290.0 & 2.4E-05 & $2.8 \mathrm{E}-05$ & 3.6E-05 \\
\hline & Tomato & $2,670.8$ & $2,936.4$ & $3,378.4$ & $2.8 \mathrm{E}-04$ & 3.4E-04 & 4.3E-04 \\
\hline
\end{tabular}

In a study in Mumbai, the level of daily dietary in spinach, radish, cauliflower, potato, apple, grapes was 195, 128.5, 122.7, 59. 8, 51.6, and 47.7 $\mu \mathrm{g} / \mathrm{kg}$, respectively. The outcomes of Bishnoi, et al. study revealed that the PAHs level in root vegetables was higher compared to leafy vegetables ${ }^{37}$, which is in agreement with the findings of the present study. In addition, leafy vegetables and fruits were more contaminated with lighter PAHs. These observations can be supported by this fact, which high volatilization and long-distance transportation capability of light PAHs lead to their deposition to places very far from the origin of pollution. Further, two- and three-ring compounds contributed to $60-85 \%$ of total PAHs since these compounds possessed high solubility of water, and favored their relatively upper uptake by these plants ${ }^{38}$.

On the other hand, the Joint Expert Committee on Food Additives (JECFA) (2005) reported an average BaP intake of $4 \mathrm{ng} / \mathrm{kg}$ b.w/day corresponding to a daily intake of $\mathbf{2 8 0} \mathrm{ng}$ per person. Based on the main food groups in the whole diet, EFSA (European Food Safety Authority) reported a median B(a)P intake in Europe of $235 \mathrm{ng} /$ person for mean dietary consumers and $389 \mathrm{ng} /$ day for high dietary consumers ${ }^{39}$. Furthermore, the higher ED values of PAH among the most consumed vegetables by Tehran citizens were in tomato (3378), potato (1251) and onion (639) $\mu \mathrm{g} / \mathrm{kg} / \mathrm{day}$. Moreover, the lower ED values of PAH among the consumed vegetables were in radish (0.5), Spinach (6) and turnip (9.4) $\mu \mathrm{g} / \mathrm{kg} / \mathrm{day}$ (Table 4). In addition, the higher ED values of PAH among the most consumed fruit items by Tehran citizens were in cucumber (4069), watermelon (1720) and kiwi (1315) $\mu \mathrm{g} / \mathrm{kg} /$ day. Further, the lower ED values of PAH among the consumed fruit items were in pomegranate (3.2), sweet lemon (12.3) and peach (13.5) $\mu \mathrm{g} / \mathrm{kg} / \mathrm{day}$ (Table 5). However, by Falco, et al. in Spain, were showed that the mean determined dietary intake of the $\triangle \mathrm{PAHs}$ was $8.4,8.2,7.4,6.3$, and $6.3 \mu \mathrm{g} /$ day for male adults, adolescents, children, seniors, and female adults, respectively 40 . 
Table 5

Uncertainty analysis for the EDi and ILCR of investigated PAH content in fruit

\begin{tabular}{|c|c|c|c|c|c|c|c|}
\hline & & \multicolumn{3}{|c|}{ EDi $(\mu g / k g / d)$} & \multicolumn{3}{|l|}{ ILCRi } \\
\hline & & $50 \%$ Perc & 75\% Perc & $95 \%$ Perc & $50 \%$ Perc & $75 \%$ Perc & $95 \%$ Perc \\
\hline \multirow[t]{5}{*}{ Citrus } & Sweet Lemon & 9.7 & 10.7 & 12.3 & 1.0E-06 & $1.2 \mathrm{E}-06$ & $1.5 \mathrm{E}-06$ \\
\hline & Orange & 571.8 & 624.5 & 710.3 & $5.9 \mathrm{E}-05$ & 7.0E-05 & $9.4 \mathrm{E}-05$ \\
\hline & Tangerine & 80.3 & 88.9 & 101.03 & $8.4 \mathrm{E}-06$ & $9.9 \mathrm{E}-06$ & 1.3E-05 \\
\hline & Grapefruit & 49.4 & 54.3 & 62.3 & 5.1E-06 & $6.1 \mathrm{E}-06$ & $7.8 \mathrm{E}-06$ \\
\hline & Lemon & 79.7 & 88.3 & 100.2 & $8.2 \mathrm{E}-06$ & $9.8 \mathrm{E}-06$ & 1.3E-05 \\
\hline \multirow[t]{9}{*}{ Fruits } & Apple & 731.7 & 808.2 & 927.5 & 7.7E-05 & $9.2 \mathrm{E}-05$ & $1.2 \mathrm{E}-04$ \\
\hline & Banana & 437.1 & 481.5 & 548.68 & $4.6 \mathrm{E}-05$ & $5.6 \mathrm{E}-05$ & 7.1E-05 \\
\hline & Kiwi & $1,031.0$ & $1,134.9$ & 1,315. 7 & $1.2 \mathrm{E}-04$ & 1.3E-04 & $1.6 \mathrm{E}-04$ \\
\hline & Pomegranate & 2.6 & 2.8 & 3.3 & $2.6 \mathrm{E}-07$ & $3.2 \mathrm{E}-07$ & 4.1E-07 \\
\hline & Peach & 10.7 & 11.8 & 13.5 & 1.1E-06 & 1.3E-06 & 1.7E-06 \\
\hline & Nectarine & 20.5 & 22.6 & 25.4 & $2.1 \mathrm{E}-06$ & $2.6 \mathrm{E}-06$ & 3.3E-06 \\
\hline & Plums & 110.9 & 121.4 & 137.4 & 1.1E-05 & $1.4 \mathrm{E}-05$ & 1.7E-05 \\
\hline & Persimmon & 47.0 & 52.0 & 59.3 & $4.9 \mathrm{E}-06$ & 5. $8 \mathrm{E}-06$ & 7.5E-06 \\
\hline & Grapes & 25.6 & 28.0 & 32.2 & 2.7E-06 & $3.2 \mathrm{E}-06$ & 4.2E-06 \\
\hline \multirow[t]{4}{*}{ Melons } & Watermelon & $1,374.2$ & $1,496.3$ & $1,721.0$ & $1.5 \mathrm{E}-04$ & 1.7E-04 & 2.2E-04 \\
\hline & Cantaloupe & 803.5 & 884.6 & $1,027.9$ & 8.3E-05 & $9.8 \mathrm{E}-05$ & 1.3E-04 \\
\hline & Melon & 146.1 & 159.9 & 181.5 & 1.5E-05 & 1.8E-05 & 2.4E-05 \\
\hline & Cucumber & $3,237.7$ & $3,537.4$ & $4,069.8$ & $3.4 \mathrm{E}-04$ & 4.0E-04 & 5.1E-04 \\
\hline
\end{tabular}

\subsection{Health risk assessment}

The exposure assessment is one of the most significant constituents of risk measurement that is applied to evaluate the probability and extent of individuals' exposure to chemical substance ${ }^{41}$. Based on the USEPA reports, $10^{-6}$ fortuity of additional human cancer over a 70 -year lifetime $\left(\right.$ ILCR $\left.=10^{-6}\right)$ is the risk considered acceptable level or the insignificant level, which is favorably comparable with the risk level of some routine activity, and the work like ${ }^{42}$.

The increased cancer risk in a lifetime is considered serious in $10^{4}$ or greater number of people $\left(\right.$ ILCR $=10^{-4}$ ). Therefore, paying attention to this health problem is of high priority. Tables 4 and 5 indicate the distribution of ILCR after 20000 iterations and with a probability of 50,75 and $95 \%$.

The mean of contributions to overall ILCR in vegetables and fruits was estimated to be 5.2E-05 and 7.7E-05, respectively, which was higher than the acceptable risk level $\left(10^{-6}\right)$. The results of a study in Spain revealed that the PAHs total daily intake is related to a $5 \mathrm{E} 10-6$ increase in cancer risk in an adult male weighing $70 \mathrm{~kg}^{40}$ compared to the findings of the present study .

According to the outcomes of the current research, the highest ILCR in the four groups of vegetables belonged to fruit (4.3E-04) and root (1.6E-04) vegetables, while the lowest was found in cabbage (3.5E-07) (Table 5 and Fig. 3 ).

Furthermore, it was found that the highest cumulative ILCR in the three groups of fruits was detected in melons (5.1E-04), followed by other fruits (1.6E-04) and then citrus (9.4E-05). Moreover, the highest ILCR in the groups of melons, citrus, other fruit, vegetables, rooted vegetables, cabbage, and leafy vegetables was related to cucumber (5.1E-04), kiwi (1.6E-04), orange (9.4E-05), tomato (4.3E-04), potato (1.6E-04), white cabbage (1.9E-06) and mixture of leek, coriander and basil (3.5E-06) (Fig. 3). Conversely, the lowest ILCR belonged to melon (2.4e-05), pomegranate (4.1e-07), sweet lemon (1.5e-06), eggplant (3.6e-05), spinach (8. 6e-07), cauliflower (3.5e-07), and radish (7.1e-08).

In addition, the most and least contribution to overall ILCR in seven groups of vegetables and fruits were related to root vegetables and melons, respectively (Fig. 4).

In a study conducted in Taiwan, the mean value of ILCRs was higher than the level of priority risk ( $\left.10^{-4}\right)$. Contrarily, the mean values of the ILRC of raw food for all people groups were in the range of $10^{-6}-10^{-5}$, which was more than the acceptable risk level $\left(10^{-6}\right)$ while lower than the priority risk level 5 .

Finally, Khillare et al. concluded that the cumulative ILCR through the vegetables dietary intake was 3.4E-06 demonstrating a slight cancer risk ${ }^{43}$.

\section{Conclusion}


Considering that the proportion of vegetables and fruits in the food basket of Iranians is relatively high and another country, it is important to test the concentration of PAHs in vegetables and fruits. Therefore, monitoring the vegetable and fruit safety in order to control the PAHs content is a priority. The present study is the first comprehensive study piloted to evaluate samples of vegetables and fruits in Iran. 192 samples from 32 types of vegetables and fruits were purchased from the Tehran market, and the level of 16 PAHs was determined using MSPE and GC/MS. The results showed that the highest PAH levels corresponded to acenaphthene $(135.1 \pm 7.1 \mu \mathrm{g} / \mathrm{kg})$ and naphthalene $(114.1 \pm 5.0 \mu \mathrm{g} / \mathrm{kg})$, whereas the lowest concentrations were those of Benzo(a)pyrene (not detected), Benzo(k)fluoranthene (not detected), Indeno(1,2,3-cd)pyrene (not detected), Benzo(b)fluoranthene (not detected) and Benzo(g,h,i)perylene (not detected). HCA and PCA were applied to evaluate the correlation between the type and amount of 16 PAHs with vegetables and fruits samples. Finally, the mean of contributions to overall incremental lifetime cancer risk in vegetables and fruits was found to be $5.2 \mathrm{E}-05$ and $7.7 \mathrm{E}-05$, which was higher than the acceptable risk level $\left(10^{-6}\right)$. Therefore, fruits and vegetables need to be routinely controlled by regulatory agencies in order to reduce their PAHs.

\section{Declarations}

\section{Acknowledgment}

This study was part of an MSc thesis of Fariba Khalili in Food Safety and Environmental Health that was conducted in laboratory of Department of Environmental Health of School of Public Health, Tehran University of Medical Sciences (TUMS).

Funding: This article does not provide any financial support.

Conflict of Interest: The authors have no conflicts of interest to declare in this work

\section{Author statement}

All persons who meet authorship criteria are listed as authors, and all authors certify that they have participated sufficiently in the work take public responsibility for the content, including participation in the concept, design, analysis, writing, or revision of the manuscript. Furthermore, each author certifies that this material or similar material has not been and will not be submitted to or published in any other publication before its appearance in the Journal of scientific reports

\section{Authorship contributions}

Nabi Shariatifar: Supervision, Design of study, Writing- Reviewing and Editing,. Fariba Khalili: Data curation, Writing- Original draft preparation, Mohammad Hadi Dehghani: Conceptualization, Visualization, . Kamyar Yaghmaeian: Writing- Reviewing and Editing Investigation,. Ramin Nabizadeh Nodehi: Saftware, Methodology. Mehdi Yaseri: Software, Validation

Best regards

Corresponding author

\section{References}

1. Vasudha Bansal and Ki-Hyun Kim, Environment international 84, 26 (2015).

2. Isabel Martorell, Gemma Perelló, Roser Martí-Cid et al., Environment international 36 (5), 424 (2010).

3. Narsi R Bishnoi, Urvashi Mehta, Umashanker Sain et al., Environmental monitoring and assessment 107 (1-3), 399 (2005).

4. Santino Orecchio, Viviana Paradiso Ciotti, and Loredana Culotta, Food and Chemical Toxicology 47 (4), 819 (2009).

5. Zhonghuan Xia, Xiaoli Duan, Weixun Qiu et al., Science of the Total Environment 408 (22), 5331 (2010).

6. Nabi Shariatifar, Manouchehr Dadgar, Yadolah Fakhri et al., Journal of Food Composition and Analysis 85, 103331 (2020).

7. Giorgia Purcaro, Sabrina Moret, and Lanfranco S Conte, Talanta 105, 292 (2013).

8. Mojtaba Moazzen, Reza Ahmadkhaniha, Mohamad Es' haghi Gorji et al., Talanta 115, 957 (2013).

9. Karsten A Fähnrich, Miloslav Pravda, and George G Guilbault, Analytical letters 35 (8), 1269 (2002).

10. Sardar Khan and Qing Cao, Journal of Soils and Sediments 12 (2), 178 (2012).

11. Eunkyung Yoon, Kyungah Park, Hyomin Lee et al., Human and Ecological Risk Assessment 13 (3), 669 (2007); Mikhail F Denissenko, Annie Pao, Moonshong Tang et al., Science 274 (5286), 430 (1996).

12. Kamila Mocek and Artur Ciemniak, Journal of Environmental Science and Health, Part B 51 (2), 96 (2016).

13. International Agency for Research on Cancer and International Agency for Research on Cancer, Overall evaluations of carcinogenicity: an updating of IARC monographs volumes 1 to 42, edited by Editor. (IARC Lyon, France:, 1987).

14. Anna Sadowska-Rociek, Magdalena Surma, and Ewa Cieślik, Environmental Science and Pollution Research 21 (2), 1326 (2014).

15. Yating Shi, Hao Wu, Chaoqiong Wang et al., Food chemistry 199, 75 (2016).

16. Amin Kiani, Mahsa Ahmadloo, Nabi Shariatifar et al., Environmental Science and Pollution Research 25 (13), 12728 (2018).

17. Amin Kouhpayeh, Mojtaba Moazzen, Gholam Reza Jahed Khaniki et al., Journal of Mazandaran University of medical sciences 26 (145), 257 (2017).

18. Mojtaba Moazzen, Amin Mousavi Khaneghah, Nabi Shariatifar et al., Arabian Journal of Chemistry 12 (4), 476 (2019).

Page $11 / 14$ 
19. Mahsa Ahmadloo, Nabi Shariatifar, Razzagh Mahmoudi et al., Journal of Mazandaran University of Medical Sciences 28 (168), 69 (2019); Mohamad Es' haghi Gorji, Reza Ahmadkhaniha, Mojtaba Moazzen et al., Food control 60, 57 (2016); Amin Kiani, Nabi Shariatifar, Saeed Shahsavari et al., Journal of Mazandaran University of Medical Sciences 29 (178), 10 (2019); Mojtaba Moazzen, Amir Hossein Mahvi, Nabi Shariatifar et al., Toxin Reviews 37 (4), 319 (2018); Mojtaba Moazzen, Noushin Rastkari, Mahmood Alimohammadi et al., Journal of Environmental Health Enginering 2 (1), 7 (2014).

20. Aliakbar Roudbari, Roshanak Rafiei Nazari, Nabi Shariatifar et al., Environmental Science and Pollution Research, 1 (2020).

21. Samane Samiee, Yadolah Fakhri, Parisa Sadighara et al., Environmental Science and Pollution Research International (2020).

22. process for conducting probabilistic USEPA. Risk assessment guidance for Superfund: volume III part A and DC: US Environmental Protection Agency risk assessment. Washington.

23. Nabi Shariatifar, Fatemeh Seilani, Behrooz Jannat et al., International Journal of Environmental Analytical Chemistry, 1 (2020).

24. Majid Arabameri, Roshanak Rafiei Nazari, Anna Abdolshahi et al., Journal of the Science of Food and Agriculture 99 (12), 5358 (2019); Ahmad Heydarieh, Majid Arabameri, Arezoo Ebrahimi et al., Journal of Chemical Health Risks 10 (2), 93 (2020).

25. G Tuteja, Chadetrik Rout, and Narsi R Bishnoi, Journal of Environmental Science and Technology 4 (6), 611 (2011).

26. AAK Abou-Arab, MAM Abou-Donia, FMSE El-Dars et al., Int J Curr Microbiol Appl Sci 3, 277 (2014).

27. Chao Chai, Qiqi Cheng, Juan Wu et al., Ecotoxicology and environmental safety 142, 181 (2017).

28. Mônica C Rojo Camargo and Maria Cecília F Toledo, Food control 14 (1), 49 (2003).

29. Yu-Na Lee, Sihyoung Lee, Jeong-Sook Kim et al., Food chemistry 277, 156 (2019).

30. Azza Zohair, Abou-Bakr Salim, Adeola A Soyibo et al., Chemosphere 63 (4), 541 (2006).

31. Soghra Bahrami, Farid Moore, and Behnam Keshavarzi, Human and Ecological Risk Assessment: An International Journal, 1 (2019).

32. Samane Samiee, Yadolah Fakhri, Parisa Sadighara et al., Environmental Science and Pollution Research, 1 (2020); K Naddafi, M Yunesian, S Faridi et al., Iranian Journal of Health and Environment 10 (1), 25 (2017).

33. Alice Paris, Jerome Ledauphin, Pauline Poinot et al., Environmental Pollution 234, 96 (2018).

34. Luís F Amato-Lourenco, Mitiko Saiki, Paulo HN Saldiva et al., Frontiers in Environmental Science 5, 77 (2017).

35. Muhammad Waqar Ashraf and A Salam, Bulletin of environmental contamination and toxicology 88 (4), 543 (2012).

36. Nabi Shariatifar, Mohammad Rezaei, Mahmood Alizadeh Sani et al., Biological Trace Element Research, 1 (2020).

37. Narsi R Bishnoi, Urvashi Mehta, and GG Pandit, (2006).

38. Lochan Singh and Tripti Agarwal, Trends in food science \& technology 79, 160 (2018).

39. Lilianne Abramsson-Zetterberg, Per Ola Darnerud, and Sören Wretling, Food and Chemical Toxicology 74, 107 (2014).

40. Gemma Falco, José L Domingo, Juan M Llobet et al., Journal of Food Protection 66 (12), 2325 (2003).

41. Fariba KHALILI, Amir Hossein MAHVI, Simin NASSERI et al., Iranian Journal of Public Health 48 (5), 902 (2019).

42. D Kofi Asante-Duah, Public health risk assessment for human exposure to chemicals, edited by Editor. (Springer, 2002).

43. PS Khillare, Darpa Saurav Jyethi, and Sayantan Sarkar, Food and Chemical Toxicology 50 (5), 1642 (2012).

\section{Figures}



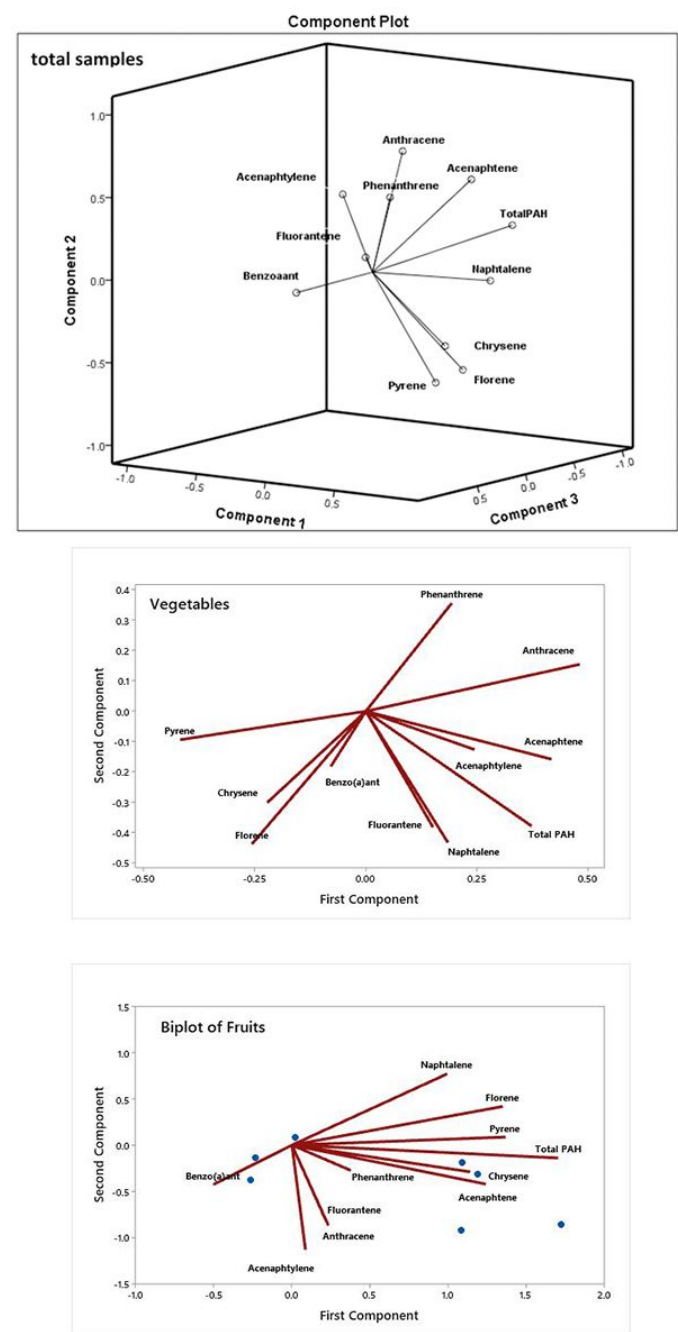

\section{Figure 1}

Principal component analysis loading plot of 16 PAHs in 32 (vegetables,fruits and total samples)
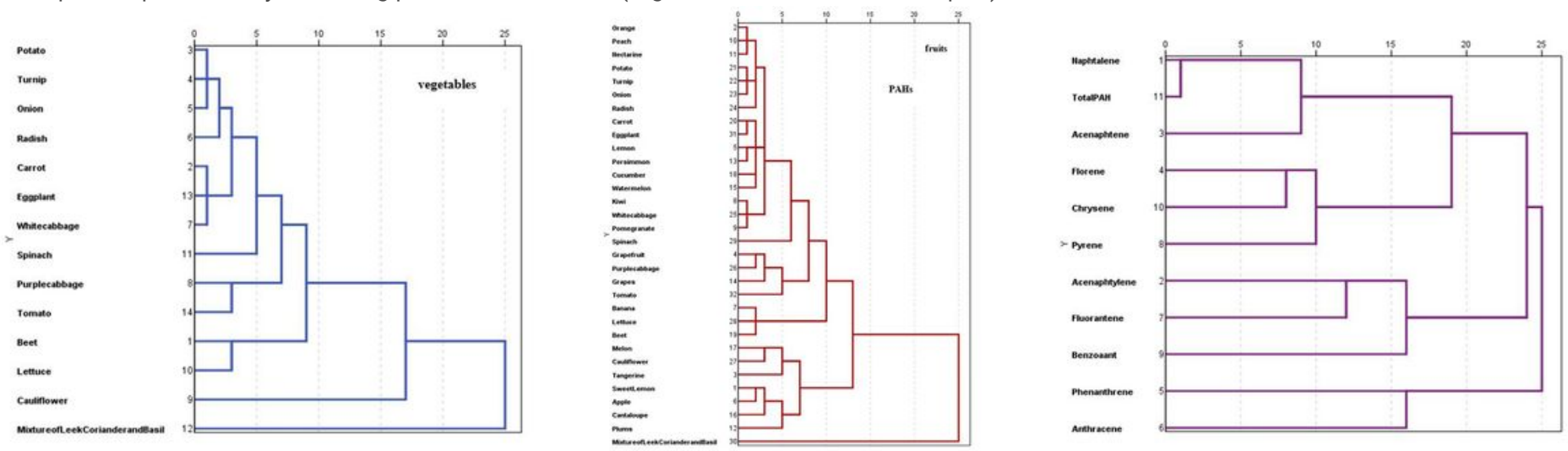

Figure 2

Hierarchical clustering results performed on the PAHs in vegetables and fruits data set. 

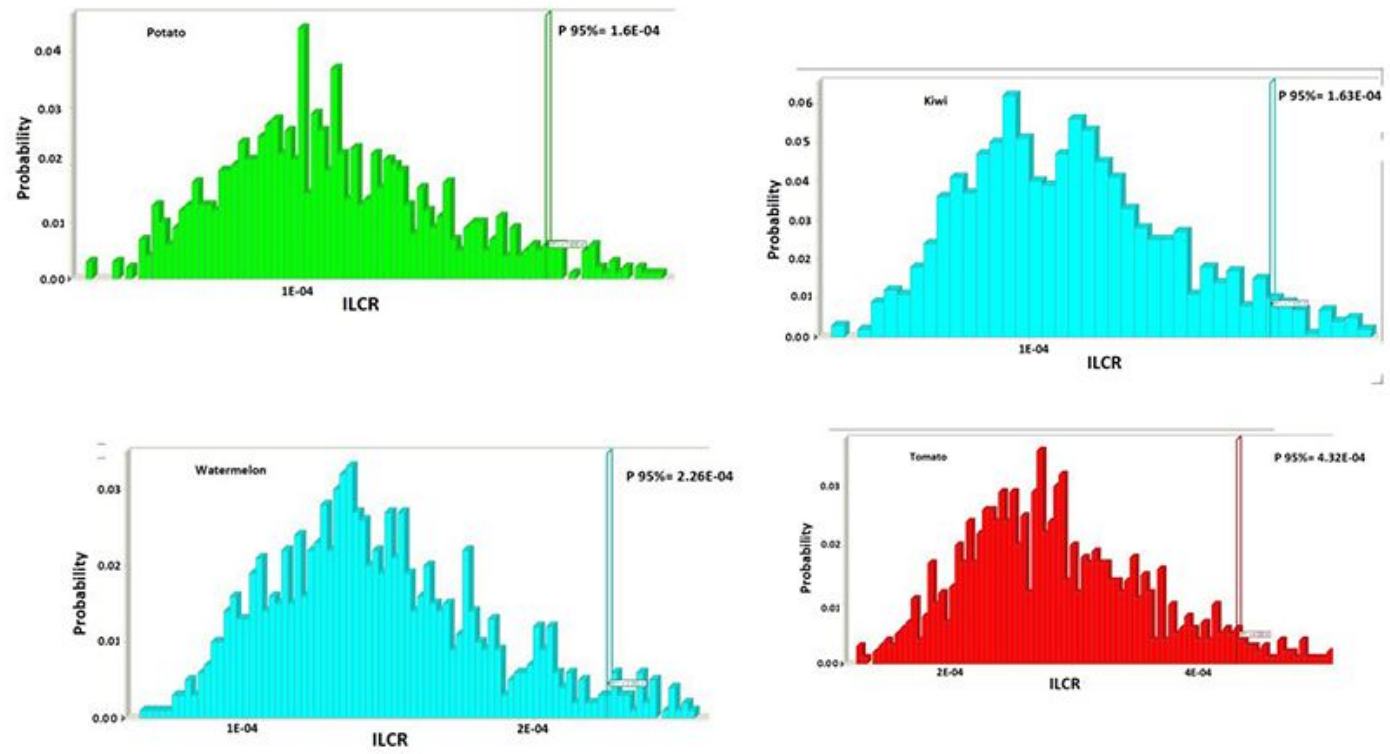

Figure 3

Simulation results for incremental lifetime cancer risk (ILCR) of PAHs in vegetables and fruits.

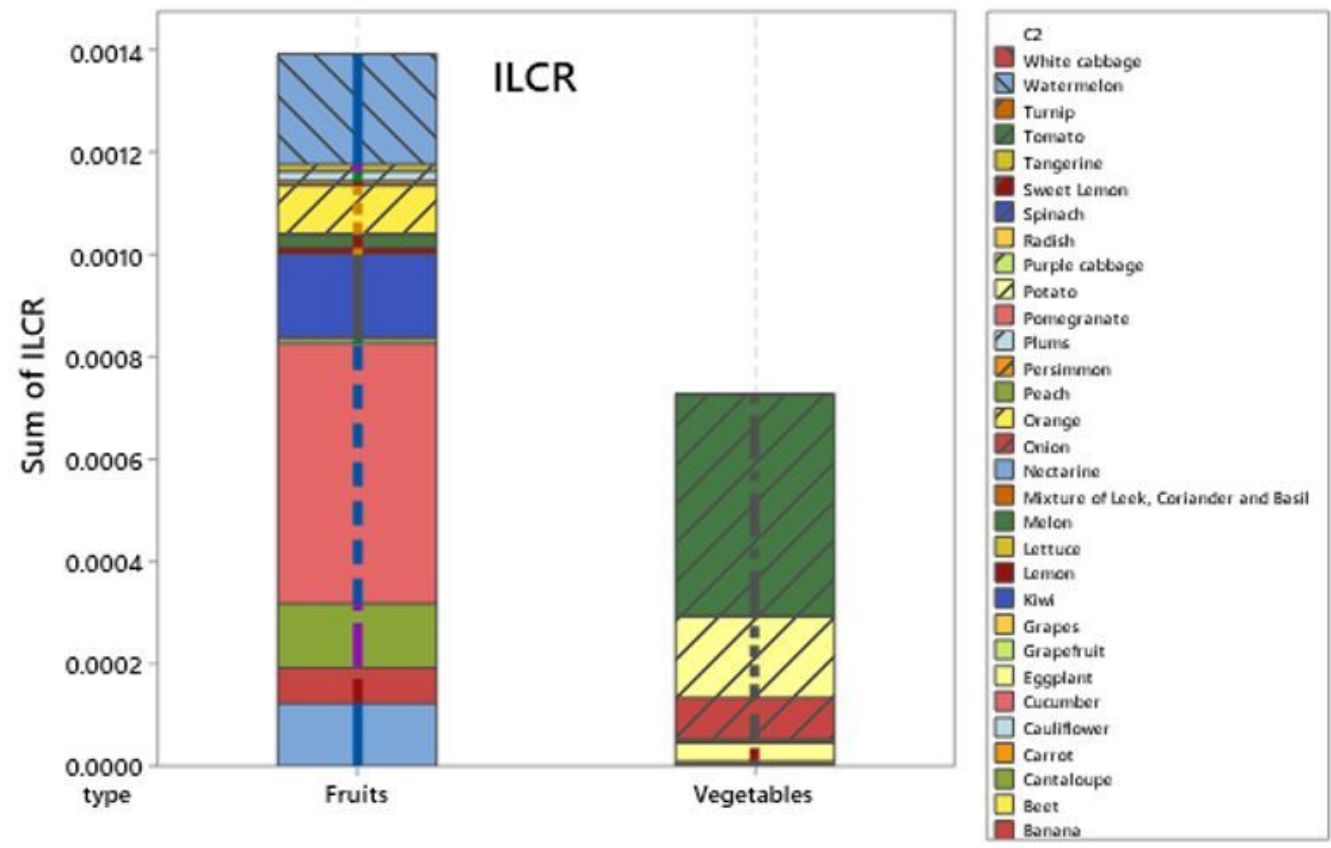

Figure 4

Comparison of the most and least contribution to overall ILCR in vegetables and fruits 